\title{
Gesture recognition algorithm design and implementation of interactive projection system
}

\author{
Chao $\mathrm{Xu}^{1}$, Yaohua Deng ${ }^{1, *}$, ,Liming $\mathrm{Wu}^{1}$,Chen $\mathrm{Xu}^{1}$, Qiaofen Zhang ${ }^{1}$
}

(1. Guangdong University of Technology; Guangdong Guangzhou 510006)

( ${ }^{*}$ Correspond author,Email: dengyaohua@gdut.edu.cn)

Key words: Interactive Projection;Gesture Recognition;Hu moment;SVM;

\begin{abstract}
With the development of multimedia computer technology , interactive projection is becoming an interactive display system and entertainment in people's daily life.Interactive projector based on actual needs, design gesture based feature extraction algorithm Hu moments, identified by SVM classification shot type, and ultimately through experiments verify the effectiveness and practicality of the system.
\end{abstract}

\section{Introduction}

Interactive projection system is a new kind of multimedia display platform.Using advanced projection display, computer vision, pattern recognition,artificial intelligence technology to create a dynamic interactive experience of fantasy ${ }^{[1]}$.

In this paper, based on the advantages of Hu moments algorithm, the method of gesture contour extraction and geometric computation based on $\mathrm{Hu}^{[2][3]}$ moments theory is developed. The improved $\mathrm{SVM}^{[4]}$ algorithm is designed to deal with the feature image and the training set is optimized to obtain the optimal solution.

\section{Gesture Recognition System Framework}

In this paper, several work of gesture recognition arrangements.We select "0" to "9" ten class static gestures as a template.Gesture pretreatment goal is to obtain a binarized image, and the outline of the gesture.Classification feature extraction stage is to select those parameters that easy classification. Choose fast and highly accurate method of classification for the system of classification trainer. Using a support vector machine classifier for gesture recognition.The overall structure diagram of gesture recognition system is shown in Fig.1.

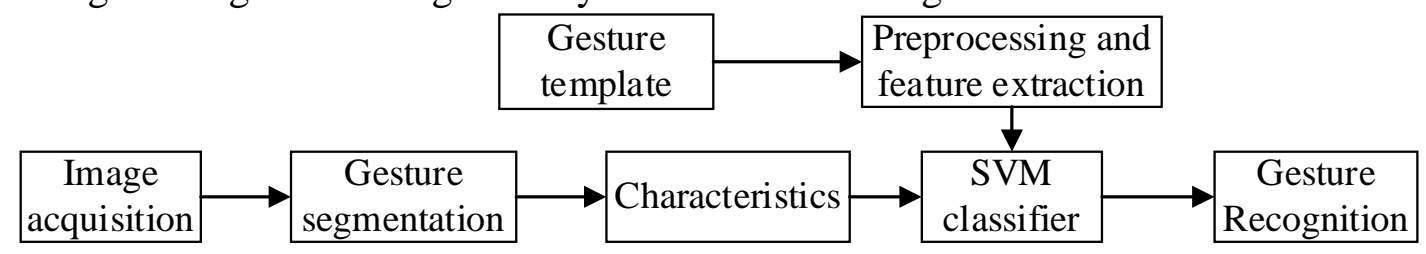

Fig.1 The structure diagram of gesture recognition system

\section{Gesture recognition system framework}

After preprocessing, the gesture images can be obtained by the binarization image, edge image and the contour matrix, which contains a lot of information to characterize the gesture. The basic task of gesture feature extraction is to select the most effective data from these data.

Gesture feature extraction based on Hu moment. The moment feature of the image range reflects the geometric properties, because in the plane of its characteristics will not change with the movement, the angle of rotation, the size of the factors, so named as the same moment. When image processing, geometric moment invariants can be used as a key feature of the object. According to this feature to image classification and other operations.

According to Hu's theory, the mathematical combination of different levels of geometric moments can be obtained by 7 characteristics. When the image motion, rotation and scale change, 
they have the characteristics of keeping the value of the same. the experiment, the video frames are collected from the static image and the Hu moment is calculated, and the average value is taken as the standard value.Because the high order moment is not only large, but also easy to be affected by noise, this paper takes the first $4 \mathrm{Hu}$ moments as the first 4 characteristic parameters M1 M4:

$M 1: \phi_{1}=\eta_{20}+\eta_{02} ; M 2: \phi_{2}=\left(\eta_{20}+\eta_{02}\right)^{2}+4 \eta_{11}{ }^{2}$;

M3: $\phi_{3}=\left(\eta_{30}-3 \eta_{12}\right)^{2}+\left(\eta_{03}-3 \eta_{21}\right)^{2} ; M 4: \phi_{4}=\left(\eta_{30}+\eta_{12}\right)^{2}+\left(\eta_{03}+\eta_{21}\right)^{2}$.

\section{Gesture recognition algorithm based on SVM}

In this paper, the basic idea of SVM is used for classification is constructed in gesture to the characteristics of the sample space, the optimal hyperplane, make gestures to the distance between sample set is the largest, best generalization ability.Algorithm idea is as follows:(1)Let the training set for the $\left\{x_{i}, y_{i}\right\}$, where $\left\{x_{i}\right\} \in R^{n}, y_{i} \in\{1,-1\}, i=1,2,3, \cdots, n$.Nonlinear function $\phi$ to achieve $\mathrm{X}$ fro $K\left(x_{i}, x_{j}\right)=\left(\phi\left(x_{i}\right) \cdot \phi\left(x_{j}\right)\right) ;(2)$ In the feature space constructed optimal hyperplane $f(x)=(w \cdot \phi(x))+b$ where $\mathrm{x}$ is the weight vector,the constraint conditions for $\max \phi(\alpha)=\frac{1}{2} \sum_{i=1} \sum_{j=1} y_{i} y_{j} a_{i} a_{j}\left(x_{i} \cdot x_{j}\right)-\sum_{j=1}^{n} a_{j}$, where $\sum_{i=1}^{n} y_{j} a_{i}=0, a_{i} \geq 0, i=1,2, \cdots, n$. To get the optimal solution $\alpha^{*}=\left(\alpha_{1}^{*}, \alpha_{2}^{*}, \cdots, \alpha_{n}^{*}\right)^{T}$;(3)Calculation: $\omega^{*}=\sum_{i=1}^{n} y_{i} a_{i}^{*} x_{i}$ and $\mathrm{m}$ the input space mapping feature space $\quad Z\left(Z \in R^{m}\right) \quad$,kernel function $\quad b^{*}=y_{i}-\sum_{i=1}^{n} y_{i} a_{i}^{*}\left(x_{i} \cdot x_{j}\right) \quad ;(4)$ Decision $\quad$ function: $f(x)=\operatorname{sgn}\left(\sum_{i=1}^{n} y_{i} a_{i}^{*} K\left(x_{i}, x_{j}\right)+b^{*}\right)$.

According to the theory of functional, as long as a function satisfies the Mercer's condition, it corresponds to the inner product of a space transformation, this function is known as the inner product function or kernel function. $\mathrm{RBF}^{[5]}$ kernel function is selected.

\section{Experimental test}

System overall program design. In this paper, the design of the algorithm flow chart of gesture recognition system is shown in Fig.2.

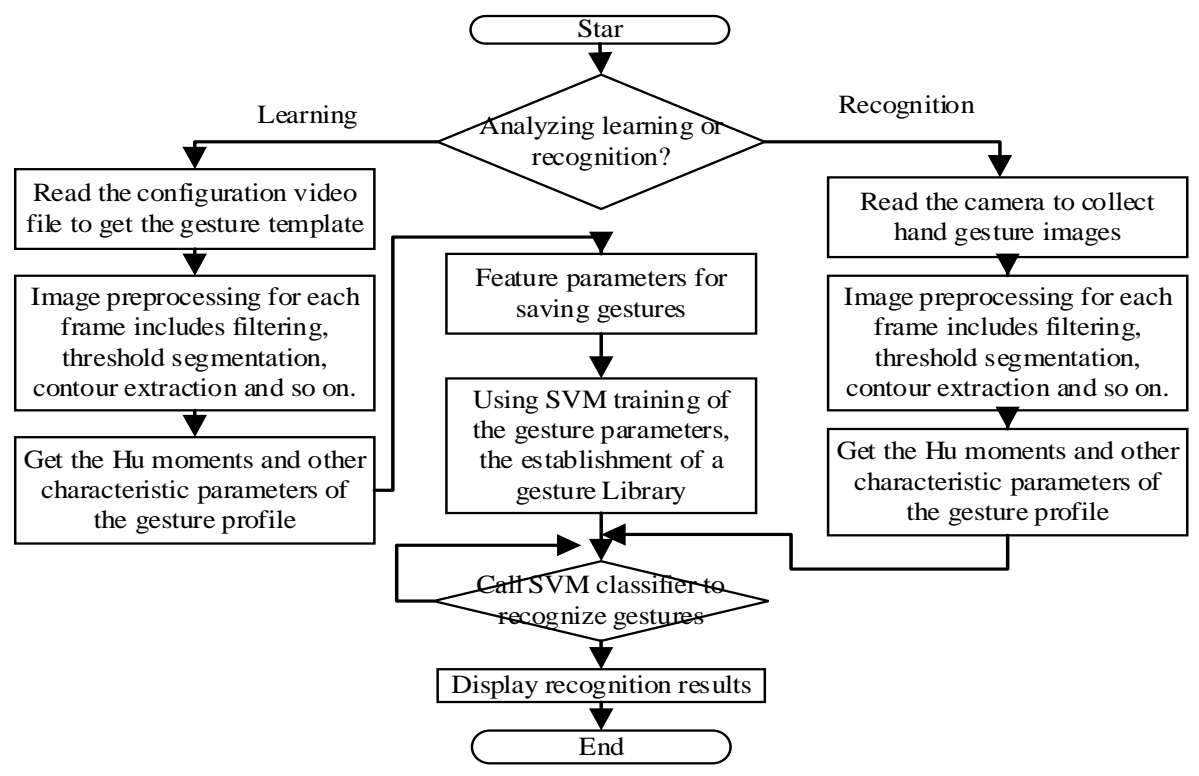

Fig.2 The design of the algorithm flow chart of gesture recognition system 
Establish sample library. Gesture recognition system based on computer vision in this paper, Firstly, the image is processed by image smoothing, color space conversion, image binarization, edge detection, contour extraction and morphological processing to get the image of binarization image, edge image and wheel profile matrix;Then, the Hu moments, perimeter area ratio and the number of depressions are calculated respectively, and the characteristic parameters of each gesture are obtained.When the sample video is trained to extract the $0 \sim 9$ gesture sample as shown in Fig.3.

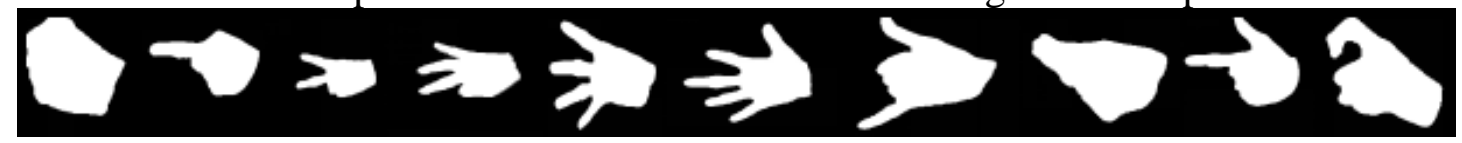

Fig.3 0 9 gesture sample

Recognition results. System through the acquisition of hand gesture samples to establish a sample library. The image of the sample is filtered, threshold segmentation and contour extraction, and the contour image is obtained, and the $\mathrm{Hu}$ moments and other characteristic parameters are obtained. Training characteristic parameters and save. The gesture image collected by the camera is processed and the parameters are extracted, then the svmPredict function is used to classify and predict. Finally, the gesture recognition is performed and the recognition result is displayed.The experiment of gesture recognition are shown in Fig.4.

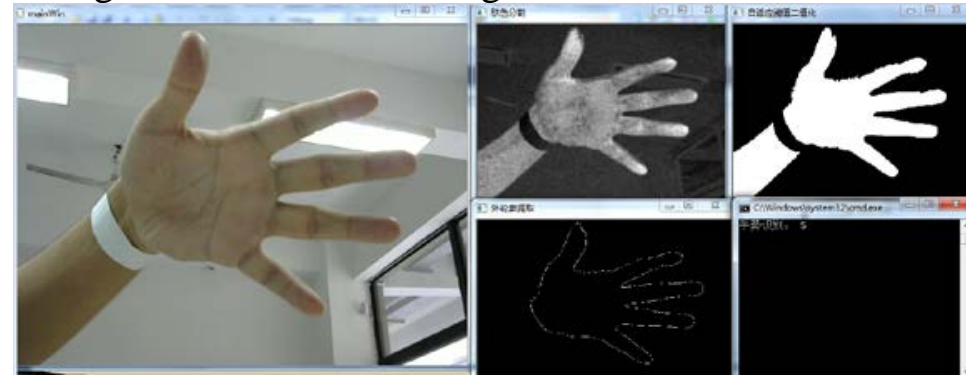

Fig.4 Gesture recognition experiment

In this paper, we have carried out hundreds of recognition to the gesture in the template library. In order to verify the geometric moment of translation, rotation and size invariance, especially in each experiment to rotate the angle of rotation, transform the distance between the gesture and the camera, changing the position of the hand in the lens.Although there are some differences between the recognition accuracy of different gestures, the experimental results show that the recognition rate of the test samples is shown in Fig.5. Correct rate is above 90\%, which can meet the needs of interactive projection system.

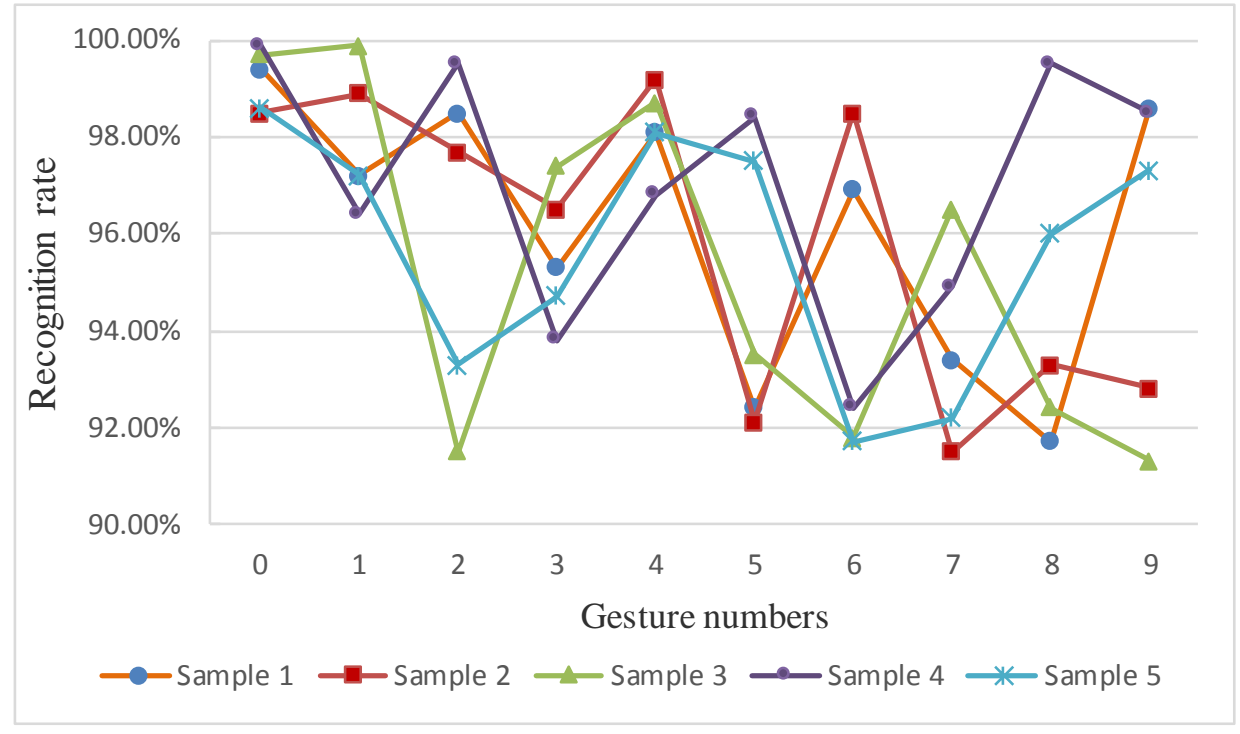

Fig.5 Gesture recognition rate of the test sample 


\section{Conclusion}

This paper designs a hand gesture recognition system based on Hu moment and SVM classifier, which is based on the study and summarization of the literature data of various kinds of hand gesture recognition technology.The framework of gesture recognition system including video file and video camera, image preprocessing module, feature extraction module, tracking module and recognition module is completed.The key is a gesture recognition system feature extraction and classification gesture recognition, feature extraction select the top four Hu moment invariants moments, gestures contour number and contour recess perimeter and area ratio as the characteristic parameter, gesture recognition algorithm selected radial SVM kernel function of the tree, and the parameters were optimized selection, to obtain a relatively high recognition accuracy and efficiency.The effect of gesture recognition can meet the needs of interactive projection system.

\section{Acknowledgement}

This work is partially supported by Science and Technology Planning Project of Guangdong Province,China (No.2013B091500046) and Special Funds for Innovative Program of Foshan, China (No.2014HT100112).

\section{References}

[1] Li Wenjie, Wu Liming, Deng Yaohua. Analysis and Discussion on the interactive technology of the projection camera man machine flexible [J]. TV technology, 2013, 37 (17).

[2] Hu M K. Visual Pattern Recognition by Moment Invariant[J].IRE Trans Information Theory, 1962,(8):179-187.

[3] Zhang Youwei, Gan Junying. Face recognition model based on invariant moment feature and neural network [J]. computer engineering and application, 2008,7.

[4] CRISTIANINI N,TAYLOR J S. An introduction to support vector machines and other kernel-based learning methods[M]. Translated by Li Guo-zheng,WANG Meng,ZENG Hua-jun.Beijing:Publishing House of Electronics Industry,2009.

[5] Louw N,Steel S J. Varibable Selection in Kernel Fisher Discriminate Analys is by Means of Recursive Feature Elimination[M]. Computational Statistics and Data Analysis, 2009,51(3)

:2043-2055. 